\title{
Extended soft-wall model for the QCD phase diagram *
}

\author{
R. ZÖllner, F. Wunderlich, B. KäMPfer
}

Helmholtz-Zentrum Dresden-Rossendorf, PF 510119, D-01314 Dresden, Germany

Institut für Theoretische Physik, TU Dresden, D-01062 Dresden, Germany

The soft-wall model, emerging as bottom-up holographic scenario anchored in the AdS/CFT correspondence, displays the disappearance of normalisable modes referring to vector mesons at a temperature $T_{\text {dis }}$ depending on the chemical potential $\mu, T_{\text {dis }}(\mu)$. We explore options for making $T_{\text {dis }}(\mu)$ consistent with the freeze-out curve $T_{\text {f.o. }}(\mu)$ from relativistic heavy-ion collisions and the cross-over curve $T_{\mathrm{c}}(\mu)$ from QCD at small values of $\mu$.

PACS numbers: $11.25 . \mathrm{Tq}, 11.10 . \mathrm{Kk}, 14.40 .-\mathrm{n}$

\section{Introduction}

In lacking still a convincing top-down approach from string theory to a proper gravity dual of QCD one must resort to bottom-up models which are designed to mimic certain wanted features of QCD. Among such approaches is the soft-wall model [1] as a particular realisation of the AdS/CFT correspondence w.r.t. the hadron spectrum, especially vector mesons. While being a phenomenological set-up, the original soft-wall model [1] can be modified to accommodate the Regge type spectrum of radial excitations of selected hadron species at vanishing temperature $T$ and chemical potential $\mu$. Extending the model further to $T>0$ one finds that, at temperatures $T \geq T_{\text {dis }}$, hadrons as normalisable states disappear [2]. It is tempting to consider such a scenario as an emulation of deconfinement. As shown in [3], one can tune the model further to achieve $T_{\text {dis }}=T_{c}$, where $T_{c} \approx 150$ $\mathrm{MeV}$ is the cross-over temperature known from lattice QCD evaluations for $2+1$ flavours with physical quark masses. There are options to let disappear all hadron states at $T_{\text {dis }}$ (instantaneous disappearance) or only the ground state, and excited states already disappeared in a narrow corridor below $T_{c}$ (sequential disappearance). For steering these details the Hawking-Page

\footnotetext{
${ }^{*}$ Presented at Critical Point and Onset of Deconfinement 2016, Wrocław.
} 
transition is a central issue.

Reference [3] focused on purely thermal effects. Here, we investigate the options for $T_{\text {dis }}(\mu)$. We provide a special modification of the soft-wall model such to make $T_{\text {dis }}(\mu)$ consistent with $T_{\text {f.o. }}(\mu)$ and $T_{c}(\mu)$, where "f.o." labels the chemical freeze-out and "c" is for the cross over. The dependence of $T_{\text {f.o. }}$ on $\mu$ is determined nowadays from hadron multiplicities observed in relativistic heavy-ion collisions at varying beam energy; system size and centrality dependencies help to consolidate the freeze-out curve $T_{\text {f.o. }}(\mu)$. The map of hadron multiplicities on the freeze-out data is provided traditionally by thermo-statistical models of the hadron resonance gas [4, 5, 6], may be supplemented by effects of inelastic, post-hadronization reactions [7]. The results are in agreement with data analyses using mean and variance of netbaryon number and net-electric charge distributions based on lattice QCD input [8]. On the other hand, lattice QCD provides ab initio calculations of $T_{c}(\mu)$, albeit restricted to a region $\mu / T<3$ due to the sign problem.

All these attempts have the goal to pin down the QCD phase diagram and to seek for a critical point that marks the onset of a curve of first-order phase transitions when going to larger values of $\mu$, realised experimentally by lowing the beam energy. Experimentally, dedicated efforts are devoted to the search for a critical point, most notably the beam energy scan at RHIC [9, 10, 11] and the program at NA61/SHINE [12, 13, 14]. Besides many models envisaging statements on the phase diagram of strong interaction matter [15, 16, 17, 18, 19, 20], also holographic approaches are to be mentioned. These aim essentially at mimicking the thermodynamics [21, 22, rather than individual hadron properties, but can address issues of deconfinement as well [23, 24]. Here, we report on the modified soft-wall model with regard of non-zero temperature and non-zero chemical potential.

\section{Modified soft-wall model}

The model pursued here is based on the action

$$
S_{V}=-\frac{1}{4 k_{V}} \int \mathrm{d} z \mathrm{~d}^{4} x \sqrt{g} e^{-\Phi(z)} F^{2}
$$

with $k_{V}$ is to be chosen to render $S_{V}$ dimensionless. The dilaton field $\Phi$ acts as a conformal symmetry breaker. The quantity $g$ denotes the determinant of the metric tensor. Equation (1) is utilised to describe the dynamics of an $U(1)$ vector field with the components $V_{M}$, where $F_{M N}=\partial_{M} V_{N}-$ $\partial_{N} V_{M}$ (indices $M, N=0, \ldots, 4$ ) is the field strength tensor, as dual to the boundary vector current, e.g. $J_{\mu} \sim \bar{q} \gamma_{\mu} q$. A special five dimensional Riemann space with coordinates $x_{0,1,2,3}$ and holographic coordinate $z$ is 
described by the infinitesimal distance squared

$$
d s^{2}=e^{A(z)}\left(f(z) d t^{2}-d \vec{x}^{2}-\frac{1}{f(z)} d z^{2}\right),
$$

where $A(z)$ is a warp function and $f(z)$ is the blackness function, both ones to be specified below. The equation of motion follows from (1), with the metric determinant to be read off (2) and $\psi=\varphi \exp \{-(A-\Phi) / 2\}$, as

$$
\left(\partial_{\xi}^{2}-\left(U_{T}-m_{n}^{2}\right)\right) \psi=0,
$$

where $\xi$ is the tortoise coordinate determined by $\mathrm{d} \xi=\mathrm{d} z / f(z)$ and $U_{T}$ is the Schrödinger equivalent potential

$$
U_{T}=\left(\frac{1}{2}\left(\frac{1}{2} \partial_{z}^{2} A-\partial_{z}^{2} \Phi\right)+\frac{1}{4}\left(\frac{1}{2} \partial_{z} A-\partial_{z} \Phi\right)^{2}\right) f^{2}+\frac{1}{4}\left(\frac{1}{2} \partial_{z} A-\partial_{z} \Phi\right) \partial_{z} f^{2} .
$$

To arrive at (3) the ansatz $V_{\mu}=\epsilon_{\mu} \varphi(z) \exp \left\{i p_{\nu} x^{\nu}\right\}$ and the gauges $V_{z}=0$ and $\partial_{\mu} V^{\mu}=0$ (Greek indices run in the range $0, \ldots, 3$ ) are employed. The normalisable solutions of (3) determine squared vector meson masses $m_{n}^{2}=$ $p_{M} p^{M}$, where $n=0$ denotes the ground state (g.s.) and $n \geq 1$ counts the radial excitations, labelled by $1^{\text {st }}, 2^{\text {nd }}$ etc.

In the spirit of [1], the soft-wall model sets a "soft wall" by the dilaton profile $\Phi(z)=(c z)^{p}$ with a scale $c$; we employ the warp factor $A(z)=\ln \left(L^{2} / z^{2}+\tilde{\mu}^{2}\right)$ with the AdS radius $L=1 / c$. Our ansatz for the blackness function is with $\vartheta\left(z_{H}\right)=\pi z_{H} T\left(z_{H}\right)-1$ (see Appendix A)

$$
f(z)=1-\frac{z^{4}}{z_{H}^{4}}\left(1+\frac{2 \vartheta\left(z_{H}\right)}{\exp \left\{\frac{2}{e} \vartheta\left(z_{H}\right)+4 \hat{\mu}^{2}\right\}}\left[\left(\frac{z}{z_{H}}\right)^{2 \exp \left\{\frac{2}{e} \vartheta\left(z_{H}\right)+4 \hat{\mu}^{2}\right\}}-1\right]\right)
$$

providing from $\left.\partial_{z} f(z)\right|_{z=z_{H}}=-4 \pi T\left(z_{H}\right)$ the Hawking temperature

$$
T\left(z_{H}\right)=\tilde{T}\left(z_{H}\right)\left(1-\hat{\mu}^{2}\right)
$$

with $\tilde{T}\left(z_{H}\right)=\tilde{T}_{\min }(1+[1 / x-2+x] / \Theta)$, where $x=z_{H} / \tilde{z}_{\min }$ and $\Theta=$ $\pi \tilde{T}_{\text {min }} \tilde{z}_{\text {min }}$. In the special case $\tilde{T}\left(z_{H}\right)=1 /\left(\pi z_{H}\right),(5)$ belongs to the metric of a Reissner-Nordström black hole embedded in an asymptotic Anti-de Sitter space. It is customary to identify $\mu=\sqrt{2} \hat{\mu} \gamma z_{H}^{-1}$ as baryo-chemical potential and $T$ as the temperature of the boundary theory. The parameter $\gamma$ arises as ratio of two coupling strengths when deriving the AdS ReissnerNordström black brane (cf. [25] and Appendix A). Equation (5) keeps the required properties of a black hole: it has a simple zero at horizon $z=z_{H}$, $f\left(z=0, z_{H}\right)=1$ and $\left(\partial_{z}^{i} f\right)_{z \rightarrow 0}=0$ for $i=1,2,3$. The above parameters 
$c, p$ and $\tilde{\mu}$ can be tuned at $T=0$ to reproduce a Regge type spectrum $m_{n}^{2}=\alpha+\beta n$ in agreement with known vector meson states forming a trajectory of radial excitations parametrised by $\alpha$ and $\beta$ [3]. Note that, for $\mu=0$, Eq. (5) facilitates numerical results agreeing on the sub percent level with those of [3].

\section{Non-zero chemical potential}

Depending on $\mu, \tilde{T}_{\text {min }}$ and $\tilde{z}_{\text {min }}, T\left(z_{H}\right)$ can display a minimum of $T_{\min }$ at $z_{\min }$ which translates into $T_{\min }(\mu)$. If so, then $(5)$ must be replaced by the trivial, non-black hole function $f=1$ for all $T<T_{\min }$, i.e. due to the Hawking-Page transition the thermal gas solution is the stable configuration. What remains is a selection of parameters $\tilde{T}_{\text {min }}, \tilde{z}_{\text {min }}$ and $\gamma$ to achieve $T_{\text {dis }}^{\text {g.s. }}(\mu) \cong T_{\text {f.o. }}(\mu) \cong T_{c}(\mu)$. We take the leading order shape

$$
T_{\text {f.o. }}(\mu) \cong T_{c}(\mu) \cong T_{0}\left(1-\kappa\left(\frac{\mu}{T_{0}}\right)^{2}+\ldots\right)
$$

with $\kappa=0.005 \ldots 0.01$ from [8] (cf. also [7) and put for simplicity $T_{0}=$ $T_{c}(\mu=0)=155 \mathrm{MeV}$ without an error band.

The dependence of $T_{\mathrm{dis}}^{\text {g.s. }}$ follows from numerical solutions of (3) with the potential (4), where the $\mu$ dependence comes from (5. 6). We employ here the parameters $p=1.99, \tilde{\mu}=0.5$ and $c=443 \mathrm{MeV}$ which provide one possible setting of a Regge trajectory with $\alpha=0.71 \mathrm{GeV}^{2}$ and $\beta=0.75 \mathrm{GeV}^{2}$ at $T=\mu=0$, as shown in [3]. The particular choice $\tilde{T}_{\min }=155 \mathrm{MeV}$ and $c \tilde{z}_{\min }=2$ is for a scenario where, for $\mu=0$, the thermal gas solution is valid for all temperatures $T<\tilde{T}_{\min }$. That is, for $T<\tilde{T}_{\min }$ the vector meson spectrum is as at $T=0$ with the implication that the thermo-statistical model analysis applies in that region with standard vacuum masses of hadrons. At $T>\tilde{T}_{\min }$, however, the black hole solution must be accomplished. Equation (3) does not allow for normalisable solutions at $T>\tilde{T}_{\min }$, i.e. just at $T=\tilde{T}_{\text {min }}$ the hadron states (here shown only for vector mesons) disappear. In such a special setting one therefore identifies both the (chiral) cross over point and the chemical freeze-out temperature at $\mu=0$ with (de)confinement. We adjust the remaining parameter $\gamma$ such to put the disappearance temperature of the ground state, $T_{\text {dis }}^{\text {g.s. }}(\mu)$ (upper dashed curve), on the freeze-out/cross-over curve (solid blue curve) in the parametrisation (7). Using the above quoted values of curvature measure $\kappa$ in the spirit of upper and lower bounds we find the results exhibited in Fig. 1. Up to a certain critical value of the chemical potential the disappearance curve of the lowest vector meson states is on the top of the freeze-out/cross-over curve for a given value of $\kappa$. The related physical interpretation is that once 

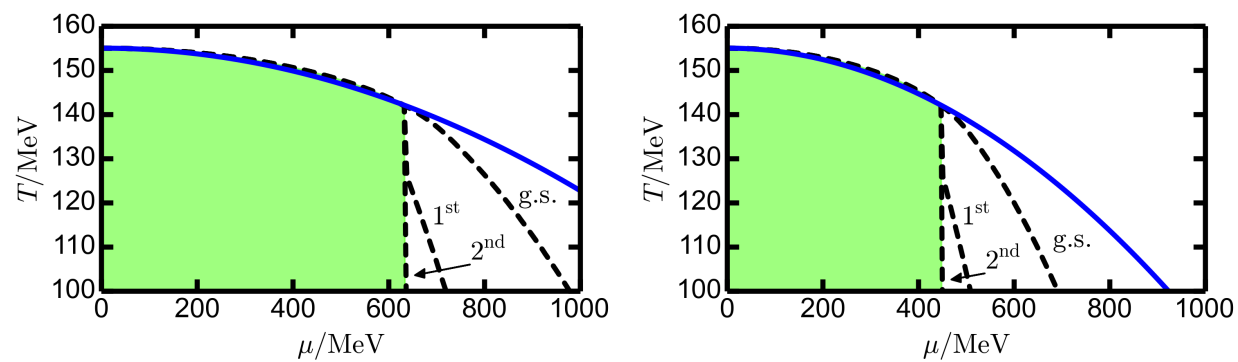

Fig. 1. QCD phase diagram with two options of the freeze-out/cross-over curve (7) (solid blue curves, left panel: $\kappa=0.005$, right panel: $\kappa=0.01$ ); note that (7) without higher-order terms holds true only in the small- $\mu$ region. In the green areas, the thermal gas solution applies. Its upper boundary is given by $T_{\min }(\mu)$. The disappearance temperatures $T_{\text {dis }}$ as a function of $\mu$ (dashed curves) of the first three vector meson states according to Eq. (3) with the potential (4) (parameters: $p=1.99, \tilde{\mu}=0.5, c=443 \mathrm{MeV}$ (cf. set 2.0 of [3]), $\tilde{T}_{\min }=155 \mathrm{MeV}, c \tilde{z}_{\min }=2$ ) are adjusted by $\gamma=7.85$ (left) and $\gamma=5.55$ (right). Up to $\mu=620 \mathrm{MeV}$ (left) or $\mu=440 \mathrm{MeV}$ (right) all states disappear instantaneously at $T=T_{\min }$. For larger values of $\mu$, where only the black-hole solution is valid (white regions), the third and all higher states do not exist at all (indicated by the vertical dashed lines); the ground state and the first excited state disappear sequentially.

a cooling piece of deconfinement matter reaches $T_{\text {dis }}^{\text {g.s. }, 1^{\text {st }}, \ldots}(\mu)$, it hadronizes by occupying statistically the available hadron states.

The above sketched scenario can be relaxed, by minor parameter variations, to have $T_{\text {dis }}^{\text {g.s. }}>T_{\text {dis }}^{1^{\text {st }}}>T_{\text {dis }}^{2^{\text {nd }}} \ldots$. i.e. a sequential appearance of vector meson states upon cooling. Figure 2 exhibits a possibility where the first two states appear sequentially in a narrow corridor centred at $T_{c}(\mu)$ for small $\mu$. If such a behaviour can be established for other hadron species too, it is still consistent with the application of the thermo-statistical models.

It is premature to extrapolate the described scenario to too large values of $\mu$, and thus to critical point issues, since (i) Eq. (7) relies on the leading-order term and (ii) lacking knowledge on $T_{c}(\mu)$, i.e. whether $T_{\text {f.o. }}(\mu) \cong T_{c}(\mu)$ at larger values of $\mu$, and (iii) unsettled options in constructing other blackness functions beyond (56).

\section{Summary}

The famous soft-wall model [1] represents a particular realisation of ideas anchored in the AdS/CFT correspondence. It can be modified to accommodate a Regge type spectrum a radial excitations of vector mesons. Con- 

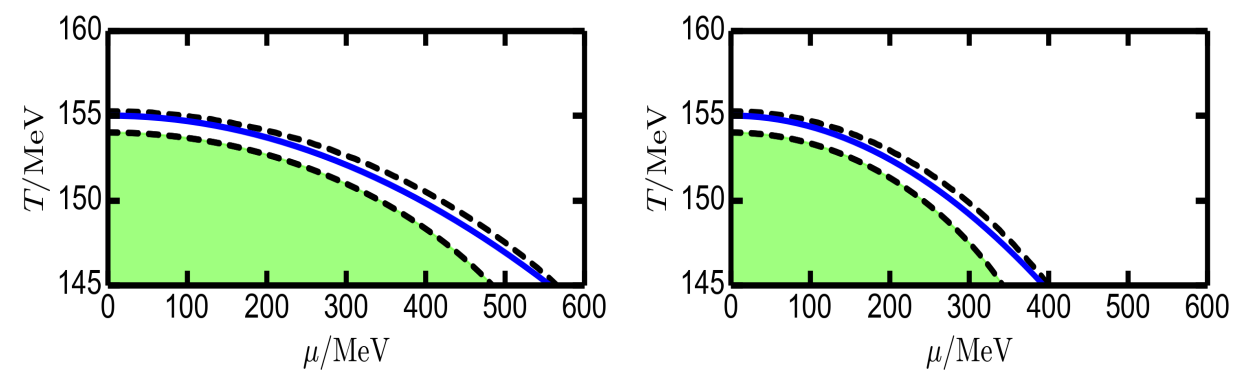

Fig. 2. As Fig. 1 but for $\tilde{T}_{\min }=154 \mathrm{MeV}, c \tilde{z}_{\min }=2.5, \gamma=8.79$ (left) and $\gamma=6.22$ (right). For all values of $\mu$ the ground state disappears at a higher temperature than the radial excitations. While the thermal gas solution is valid, all excited states disappear instantaneously.

sidering vector mesons as prototypical representatives of hadrons one can further modify such a gravity field duality model to study the fate of certain hadron species immersed in a hot and dense ambient medium. Parameters can be tuned to let disappear vector mesons as normalisable modes above a temperature to be identified tentatively with "deconfinement temperature" or, more specifically, with the chiral cross-over temperature $T_{c}[3]$, thus extending the approach in [2]. Following, e.g. [8] (see also [26]) in identifying the chemical potential dependence of $T_{c}(\mu)$ with the freeze-out systematics found from heavy-ion experiments and condensed in $T_{\text {f.o. }}(\mu)$ at small $\mu$, we have demonstrated that the suitably adopted soft-wall model allows for a consistent scenario: Once a temperature $T_{\text {dis }}$ is reached upon cooling of a piece of "deconfined matter", hadrons appear, either suddenly at once or sequentially in a narrow corridor of temperatures, and are ready for statistical distribution.

\section{Appendix A}

The goal is to extend the black-hole function in AdS, $f_{\mathrm{BH}}(z)=1-$ $\left(z / z_{H}\right)^{4}$, yielding $T_{\mathrm{BH}}\left(z_{H}\right)=1 /\left(\pi z_{H}\right)$, and the Reissner-Nordström blackhole function, $f_{\mathrm{RN}}(z)=1-\left(1+\frac{1}{2} \hat{\mu}^{2}\right)\left(z / z_{H}\right)^{4}+\frac{1}{2} \hat{\mu}^{2}\left(z / z_{H}\right)^{6}$ in AdS, yielding $T_{\mathrm{RN}}\left(z_{H}\right)=\left(\pi z_{H}\right)^{-1}\left(1-\hat{\mu}^{2}\right)$ [25]. Clearly, $f_{\mathrm{RN}}\left(z ; z_{H}, \hat{\mu}=0\right)=f_{\mathrm{BH}}\left(z ; z_{H}\right)$. As in [3] we start from the general statement that for all positive $i$ with $i>4\left(\pi z_{H} T\left(z_{H}\right)-1\right)=: 4 r$ the function $f$ defined by

$$
f(z)=1-\frac{z^{4}}{z_{H}^{4}}\left(1+\frac{4 r}{i}\left(\frac{z^{i}}{z_{H}^{i}}-1\right)\right)
$$


is a suitable blackness function, i.e. $f\left(z=0, z_{H}\right)=1,\left(\partial_{z}^{i} f\right)_{z \rightarrow 0}=0$ for $i=1,2,3$ and the simple zero at the horizon, $f\left(z=z_{H} ; z_{H}\right)=0$. To recover the Reissner-Nordström case, we observe that $r=-\hat{\mu}^{2}$ and $i=2$ are required. To construct a proper blackness function we can apply any function $h: \mathbb{R} \rightarrow \mathbb{R}$ with $h$ positive, $h(x)>x$ for all $x \in \mathbb{R}$ and $h\left(-4 \hat{\mu}^{2}\right)=2$ and set $i=h(4 r)$. One possibility is $h(x)=2 e^{a x+4 \hat{\mu}^{2}}$ for all $a \geq 1 / 2 e$ which yields (5) for $a=1 / 2 e$.

\section{REFERENCES}

[1] A. Karch et al., Phys. Rev. D 74, 015005 (2006).

[2] P. Colangelo, F. Giannuzzi, S. Nicotri, JHEP 1205, 076 (2012).

[3] R. Zöllner, B. Kämpfer, arXiv:1607.01512v1 [hep-ph] (2016).

[4] A. Andronic, P. Braun-Munzinger, J. Stachel, Nucl. Phys. A 834, 237 (2010).

[5] J. Cleymans et al., Phys. Rev. C 71, 054901 (2005).

[6] F. Becattini et al., Phys. Rev. C 85, 044921 (2012).

[7] F. Becattini et al., arXiv:1605.09694 [nucl-th] (2016).

[8] A. Bazavov et. al, Phys. Rev. D 93, 014512 (2016).

[9] A. Adare et al., Phys. Rev. Lett. 109, 152301 (2012).

[10] L. Adamczyk et al., Phys. Rev. C 88, 014902 (2013).

[11] D. McDonald, EPJ Web Conf. 95, 01009 (2015).

[12] M. Gazdzicki, Eur. Phys. J. ST 155, 37 (2008).

[13] T. Czopowicz, arXiv:1503.01619 [nucl-ex] (2015).

[14] A. Aduszkiewicz et al., arXiv:1510.00163 [hep-ex] (2015).

[15] F. Wunderlich, R. Yaresko, B. Kämpfer, J. Mod. Phys. 7, 852 (2016).

[16] R. Stiele, J. Schaffner-Bielich, Phys. Rev. D 93, 094014 (2016).

[17] T. Herbst, J. Pawlowski, B.-J. Schaefer, Phys. Rev. D 88, 014007 (2013).

[18] B.-J. Schaefer, J. Pawlowski, J. Wambach, Phys. Rev. D 76, 074023 (2007).

[19] B.-J. Schaefer, M. Wagner, Phys. Rev. D 79, 014018 (2009).

[20] O. Scavenius et al., Phys. Rev. C 64, 045202 (2001).

[21] O. de Wolfe, S. Gubser, C. Rosen, Phys. Rev. D 83, 086005 (2011).

[22] S. Finazzo, R. Rougemont, H. Marrochio, J. Noronha, JHEP 1502, 051 (2015).

[23] U. Gürsoy, E. Kiritsis, JHEP 0802, 032 (2008).

[24] U. Gürsoy, E. Kiritsis, F. Nitti, JHEP 0802, 019 (2008).

[25] M. Ammon, J. Erdmenger, Gauge/Gravity duality, Cambridge University Press (2015).

[26] P. Braun-Munzinger, J. Stachel, C. Wetterich, Phys. Lett. B 596, 61 (2004). 